BNL-108137-2015-JA

\title{
WILEY-VCH
}

DOI: 10.1002/ ((ADFM.201502463R1))

Article type: Full Paper

\section{Long Range Self-assembly of Polythiophene Breath Figures: Optical and Morphological Characterization}

Prahlad K. Routh ${ }^{1,2}$, Dmytro Nykypanchuk ${ }^{1}$, T. A. Venkatesh ${ }^{2 *}$ and Mircea Cotlet ${ }^{1,2 *}$

Prahlad K. Routh, Dr. Dmytro Nykypanchuk and Dr. Mircea Cotlet, ${ }^{1}$ Center for Functional Nanomaterials, Brookhaven National Laboratory, Upton, NY 11793 , E-mail: cotlet@bnl.gov

Dr. T.A. Venkatesh

${ }^{2}$ Department of Materials Science and Engineering, Stony Brook University, Stony Brook, NY 11790 .

E-mail: t.venkatesh@ stonybrook.edu

Keywords: breath figure technique, conjugated polymers, light harvesting, semitransparent thin film, crystallinity

Abstract. Large area, device relevant sized microporous thin films were formed with commercially available polythiophenes by the breath figure technique, a water-assisted micro patterning method, with such semitransparent thin films exhibiting periodicity and uniformity dictated by the length of the polymer side chain. Compared to drop casted thin films, the microporous thin films exhibit increased crystallinity due to stronger packing of the polymer inside the honeycomb frame.

\section{Introduction}

Ordered microporous polymer films have gained increased attention in the past several years as they are being explored for potential applications in soft lithography ${ }^{[1]}$, tissue engineering ${ }^{[2]}$, catalysis $^{[3]}$, and as superhydrophobic surfaces ${ }^{[4]}$. The regularity of pore size and the long-range order is the most sought out feature for many of these applications and this has been a long 


\section{WILEY-VCH}

standing challenge with the Breath Figure Technique $(\mathrm{BFT})^{[5]}$. There are a variety of bottom up and top down patterning techniques such as photolithography, electron beam lithography, chemical etching, and printing techniques for producing ordered microstructures ${ }^{[6]}$. Although the versatility of structures produced by lithographic techniques is large, the initial cost of equipment and the needed skilled labor is high. Hence there has been a stress recently on low cost techniques to produce highly ordered structures with tunable pore size using self-assembly ${ }^{[7]}$. BFT is one such method, which is driven by water based self-assembly. In BFT, a polymer is dissolved in a low boiling point solvent and casted on a substrate in high humidity atmosphere. Spontaneous evaporation of the volatile solvent leads to a decrease in temperature of the top surface of the solution $\left(-6 \text { to } 0^{\circ} \mathrm{C}\right)^{[8]}$. As a result of this temperature drop, small water droplets condense on the surface of the solution. These micro-droplets grow with time $\left(D \sim t^{1 / 3}, D\right.$, diameter, t, time) but when they come in contact with another droplet, they do not tend to coalesce $^{[9]}$. The polymer precipitates between these water micro-spheres, preventing them from coalescing with each other. As a result, these uniform sized micro-spheres of water droplets arrange themselves in a hexagonally packed pattern on the top of solution. After solvent evaporation and subsequent evaporation of water, a thin polymer film is left with the imprint of cavities created by the hexagonally arranged micro-spheres. Such pattern is usually referred to as a honeycomb pattern. The long-range order and regularity of honeycomb patterns depend on various experimental parameters such as humidity, molecular weight of polymer, solvent, polymer concentration, temperature and the nature of the substrate used for deposition ${ }^{[10]}$. These parameters control the evaporation rate of the solvent, prevent coalescing of water droplets and affect the honeycomb final film morphology. The effects of these experimental parameters were summarized in several review papers ${ }^{[11]}$. The dynamics of this seemingly simple method is rather complex due to the Marangoni convection currents generated by temperature gradient ${ }^{[8,}{ }^{12]}$. 


\section{WILEY-VCH}

Hence the apparatus deposition design and the precise control of these experimental parameters become extremely important for achieving reproducibility of ordered honeycomb patterns. Several mechanisms have been proposed to explain the heterogeneity seen in the BFT process and it is also believed that there could be more than one mechanism at interplay during the breath figure Process.

There has been a recurring debate as to which polymer type can form honeycomb patterns by $\mathrm{BFT}^{[11 a]}$. Initially it was believed that only polymers with a star shape could form such micro structures $^{[13]}$, later on honeycomb films were obtained with polystyrene polymers with linear structure and it was proposed that the viscosity of the solution is critical in forming honeycomb patterns ${ }^{[14]}$. Since then honeycomb patterns have been demonstrated with block-copolymers, amphiphilic copolymers, hyper-branched polymers, organic/inorganic hybrid materials. ${ }^{[15]}$ Recently BFT has been demonstrated with non-aqueous vapors ${ }^{[16]}$ and on non-planar substrates ${ }^{[17]}$. It is believed that some polymer architectures provide a robust set of process parameters which can allow tuning of the honeycomb pore structure, while other polymer architectures provide a narrow range of process conditions which can produce honeycomb morphology, hence making reproducibility difficult.

Honeycomb patterns have been also demonstrated with conductive polymers ${ }^{[18]}$ and such microporous thin films have been recognized for their potential as building blocks for interesting optoelectronic device application areas such as sensing and transparent photovoltaics. However, prior studies in the field have relied largely on custom synthesized conjugated polymers for creating relatively smaller area honeycombs. Furthermore, the relationships between the processing conditions, polymer chemistry and honeycomb structure formation and their resultant optoelectronic properties have not been fully understood. 


\section{WILEY-VCH}

Hence, in this present study we focused on developing a BFT method where several important process conditions such as temperature, humidity and air flow can be controlled and applied this BFT to a series of commercially available polythiophene (PT) derivatives which differ by the side chain length (Scheme 1). These polymers have been previously investigated as organic photovoltaic candidate materials. ${ }^{[19]}$ To compare the honeycomb formation capability of the four PT derivatives vs side chain effect, we have kept processing parameters like regioregularity, concentration, molecular weight, substrate chemistry and evaporation rate fixed and then studied the effect of increasing side chain on breath figure forming capability.

We have been able to demonstrate highly ordered microporous thin films with large surface area $\left(\sim 5 \times 5 \mathrm{~mm}^{2}\right)$, the largest size reported so far for conjugated polymer honeycomb thin films, an achievement making such semi-transparent films suitable candidates for sensing and semitransparent photovoltaic applications.

\section{Results}

\subsection{Side Chain effect on Ordering of Pores.}

In this study we used a series of commercial polythiophene conjugated polymers where the alkyl side chain length is varied from $m=6$ (poly (3-hexyl thiophene) or P3HT), to $m=8$ (poly (3-octyl thiophene) or P3OT), to $\mathrm{m}=10$ (poly (3-decyl thiophene) or P3DT) and finally to $\mathrm{m}=12$ (poly (3dodecyl thiophene) or P3DDT) (Scheme 1). These polymers, commercially available from Rieke Metals, were dissolved at similar concentrations in CS2 and were subjected to the same processing conditions in a BFT apparatus described in detail in Supporting Information (SI, Figure S1) in order to obtain honeycomb films. While BFT films were deposited on various substrates like glass, hydrophilic glass, ITO, $\mathrm{MoO}_{3}$ and $\mathrm{ZnO}$ coated glass, defect free breath figure films were formed mainly on glass coated with oxides, in particular $\mathrm{MoO}_{3}$, for which all characterization reported herein applies. 


\section{WILEY-VCH}

Figure 1 shows optical micrographs of the PT-based honeycombs, both at low and high magnification, for all four polymers, demonstrating large area ordered honeycombs. Figure 2 are Scanning Electron Microscopy (SEM) images of PT-based honeycombs along with Fast-Fourier Transform (FFT) patterns obtained by image processing (see SI for details). The hexagonal packing and long-range order can be deduced from such FFT patterns. P3OT shows long range order along with perfect 2D hexagonal ordering. A photograph of a P3OT honeycomb taken with an ordinary CC camera shown in SI, Figure S2, further demonstrates the long-range order of the BFT made thin films. In such films, areas as large as $\sim 5 \times 5 \mathrm{~mm}^{2}$ in size with highly regular honeycomb patterns are present, a size that is sufficient for future transitions of such films in sensory and photovoltaic devices. For the other PT derivatives, although they showed short range order, the presence of diffused rings in their associated FFT pattern (Fig.2) indicated the presence of 2D disorder in the honeycomb film at large scale.

For P3OT we found favorable processing conditions to be in a wide-range of humidity and airflow rate values when compared to $\mathrm{P} 3 \mathrm{HT}$ which had a rather high tendency to coalesce. Processing conditions for P3DT and P3DDT were found to be more robust compared to P3HT but the order of honeycombs films and reproducibility from these polymers were not on par with P3OT. In order to make polymer solutions amenable for honeycomb formation, we needed to age them for at least 7 days at room temperature, in the dark. Except for P3HT, prolonged ageing of polymer solution leads to improved flexibility in processing conditions, including formation of thinner honeycomb films by the use of lower polymer concentration. ${ }^{[18 b]}$

\subsection{Pore Size Distribution and Order Parameter from Voronoi Tesselation}

Pore sizes were found to vary with side chain length as observed in Figure 3. The longer the side chain, the smaller the pore size when these honeycomb were processed in similar conditions 


\section{WILEY-VCH}

including concentration. Details on pore size estimation are given in SI. It's worth noting that, there is a radial variation of pore size in such large size films due to changes in the evaporation rate from the edge to the center of the film, with the pore size increasing from edge to center (see Figure S3, SI).

One simple way to visualize the ordering of pores in two dimensions is by the use of Voronoi Tesselation (VT) method, a popular approach in computational geometry for modeling spatial structures, for pattern recognition and for localization optimization ${ }^{[20]}$. In VT, a plane with $n$ points is divided into convex polygons such as each polygon contains exactly one generating point and the vertices of each cell is equally spaced to its generating points. Given the input of the centroid of pores of the honeycomb, the VT method provides a graphical representation of nearest neighbors. The number of edges from each cell can be used to get the probability of having 6 nearest neighbors and the hexagonal packing can be then compared ${ }^{[21]}$. Also, with the probability of all possible nearest neighbors $(\mathrm{NN})$, an order parameter (entropy, S) defined below can be used to assign each image in Figure 2.

$$
S=-\sum_{i=3}^{10} p_{i} \cdot \ln p_{i}
$$

Here $p_{i}$ is the probability of finding $i$ number of nearest neighbors. The smaller the order parameter $\mathrm{S}$ is, the more order the system exhibits. The observed variation of order parameter with side chain length (Figure 4b) and the probability of nearest neighbors (Figure 4a), both support the high hexagonal ordering seen in $\mathrm{P} 3 \mathrm{OT}$ honeycomb films when compared to the rest of the PT-based honeycombs.

\subsection{Crystalline vs Amorphous-like Phase in PT-based Honeycomb Films}

Fluorescence emitted by a conjugated polymer is a property influenced by the extent of the $\pi$ conjugation system of the polymer backbone, and this can provide information on the polymer chain conformation or aggregation state or changes of such properties since such changes are 


\section{WILEY-VCH}

usually accompanied by intra- or inter-chain quenching by energy transfer. ${ }^{[22]}$ Figures $5 \mathrm{a}-\mathrm{d}$ are confocal fluorescence lifetime microscopy (FLIM) images of honeycomb thin films process by BFT for the four PTs and Figures 5i-1 are spatially resolved, confocal micro-photoluminescence (PL) spectra measured at the honeycomb frame (black color) and hole (green color) from each of the PT-based honeycomb this films. As a comparison, Figure S2, SI, displays micro-PL spectra from the four PTs in solution and drop casted thin films. A detailed analysis of the micro-PL spectra and FLIM images in Figure 5 helps us to understand the heterogeneity in polymer aggregation state within (i) a given honeycomb and (ii) among various types of PT-based honeycombs which, as explained below, we can associate with (i) differences in polymer packing across the honeycomb framework and with (ii) differences in crystallinity among the four types of PTs and resulting from differences in side chain length. Table 1 includes PL lifetimes (amplitude averaged values, see SI for details) estimated from decays measured from diffractionlimited spots in the honeycomb frame and hole, and for a comparison from solution and dropcasted thin films for all four PTs. Freshly prepared polymer solutions exhibit PL lifetimes of about 0.66ns which are rather long for PTs and which indicate $\mathrm{CS}_{2}$ is a good solvent for all four $\mathrm{PTs}^{[22 \mathrm{~b}]}$. Ageing (over 7-days) decreases the PL lifetime to about $0.58 \mathrm{~ns}$ for all four PTs, most probably due to formation of self-quenched aggregates. ${ }^{[23]}$ PL spectra from either fresh or aged solutions are broad, with a single peak at around 590nm (Figure S3, SI). Drop casting any of the four PTs produces thin films with red shifted, bi-peak PL spectra with peaks @650nm and $725 \mathrm{~nm}$, previously assigned to vibronic, $0-0$ and $0-1$ transitions, respectively ${ }^{[24]}$. The ratio of these PL peaks in drop casted films estimated from the micro-PL spectra from Figure S3, SI as $\mathrm{R}=\mathrm{PL} @ 650 / \mathrm{PL} @ 725$ is in the range 1-1.1, e.g R $\geq 1$ (see Table 1) which is common for 0-0 and 01 vibronic transitions associated with PL from aromatic molecules. PL lifetimes from drop casted films increase in value with the increase in side-chain length (Table 1, i.e. from P3HT to P3DDT) 


\section{WILEY-VCH}

and an explanation for this phenomenon is given below. PT polymers including those studied here prefer to self-pack in solid phase in ordered, e.g. crystalline-like aggregates because of the strong $\pi-\pi$ stacking of polymer backbones. $\pi-\pi$ stacking promotes inter-chain quenching which in turn red shifts the PL spectrum in film compared to solution and decreases both the PL quantum yield and PL lifetime ${ }^{[25]}$. An increase in polymer side chain length decreases $\pi$ - $\pi$ stacking interaction and this in turn decreases inter-chain quenching, leading to recovery (increase) of PL, including PL lifetime ${ }^{[26]}$ (Table 1).

FLIM images from honeycomb PTs feature clear differences in PL lifetimes in the frame and in the holes (Fig.5a-d, insets, Table 1). Similarly, there are differences in the PL spectra measured in the frame and in the hole (Fig.5i-1, black vs green colored spectra). For P3HT, the PL spectrum measured in the frame is similar in shape to the drop cast film PL spectrum (Fig.5i, black vs Fig.S4,SI), with a ratio of the high and low energy peaks $\mathrm{R}=1.1$ and with these peaks spectrally shifted @ 660nm and 710nm, while the PL spectrum measured in the hole is broad, vibrationless, and single peaking @710nm (Fig5i). For P3OT, the PL spectrum measured in the frame is still bi-peak, with an R>1, however, the PL spectrum from hole, while remaining bi-peak, now has a $\mathrm{R}<1$ (Fig.5j). For P3DT and P3DDT the PL spectra in the frame are still bi-peak, but with an $\mathrm{R}<1$, while the PL spectra in the hole are single (@720nm) and bi-peak, respectively (Figs.5k\&l). The observation of single peak (@705-715nm range) and bi-peak (@650nm and @705-715nm range) features in the PL spectra from PT honeycombs and of $R<1$ values strongly suggests two polymer aggregation states are present in honeycomb films, amorphous-like disordered aggregate state and crystalline state ${ }^{[27]}$, whose contribution dictates the ratio $\mathrm{R}$. This in turn reflects the presence of heterogeneity in crystallinity in PT honeycomb thin films. The polymer crystalline phase is similar to that usually observed in drop casted films (Fig.S4, SI), with a bi-peak spectrum with $0-0$ and $0-1$ vibronic transitions (@650nm and 725nm, respectively), while the 


\section{WILEY-VCH}

amorphous-like phase has a broad, single peak at around 705-710nm. Depending on the contribution of the two phases at a given place in the honeycomb film, the resulting spectrum might be bi-peak or single peak. For example, for P3HT, P3OT and P3DT, the crystalline phase dominates in the frame, while the amorphous-like phase dominates in the hole. For P3DDT, the crystalline phase dominates both in frame and hole, since this framework shows rather large thickness in the hole regions, (i.e., holes are not deep). We believe these differences in crystallinity arise from the different evaporation speeds of the solvent in the frame and in the holes which are imposed by BFT. Honeycomb frame is formed between adjacent water droplets which force a large amount of polymer material together to form a thick $(\sim 1 \mu \mathrm{m})$ film following solvent and water evaporation. This provides evaporation rates similar to drop casting, permitting the polymer to pack and form crystalline domains. In holes, solvent evaporation is faster because of less polymer material and as such the polymer has no time to crystalize, forming an amorphous phase. The idea of the presence of both crystalline and amorphous phase in PT honeycombs is also supported by the PL lifetimes observed in the frame and the hole, with PL lifetimes increasingly quenched in the latter case as one would expect from a self-quenched, red shifted aggregate.

P3OT is an exception, showing opposite behavior with respect to PL lifetimes of frame and holes and we may relate this with the high tendency of this particular polymer to form crystalline domains even in holes, at low concentration of polymer. Small and Wide Angle X-ray data (WAXS) recorded from PT-based honeycomb and drop cast films support our hypothesis, indicating increased crystallinity in honeycomb sample compared to drop cast film, and this increase in crystallinity is more pronounced in the case of P3OT polymer (See Figure 6 and Figure S5, SI and details on crystallinity estimation by SAXS/WAXS).

\section{Discussion}




\section{WILEY-VCH}

BFT has been demonstrated as a versatile, cost-effective method for producing ordered microporous structures with tunable pore size and frame thickness obtained through changes in processing parameters such as humidity, concentration of solution, temperature of substrate or airflow rate ${ }^{[18 b]}$. The effect of these individual parameters has been evaluated extensively by many groups and on different type of polymers ${ }^{[11 \mathrm{~d}]}$. Most of the BFT setups reported so far are very simple in nature and do not offer a precise reproduction of the morphology in the films due to inherent non-equilibrium nature of the evaporation process in the breath figure processing. BFT method is also susceptible to environmental factors when using non-PS based polymers, which provide less robust processing conditions to produce stabilized water droplets. Hence, in order to control the external environmental factors we designed a controlled humidity chamber where PT solutions were casted directly on substrates (Fig.S1, SI). At constant humidity, the evaporation rate of the solution is affected by the airflow rate and by the surface temperature of the substrate. Controlling the flow rate is crucial and having a laminar flow helps obtain large area honeycomb thin films. With the same experimental setup, we found possible to vary a whole range of parameters to explore the optimum parameters for achieving the best structures possible for a given polymer solution.

As shown by the SEM images in Figure 1, all four polymers are able to form honeycomb films by BFT after ageing, however, P3OT excels in hexagonal ordering and long-range (large area) thin film with hexagonally arranged porous structure as suggested by Voronoi Tessselation. P3OT polymer also provides more robust processing conditions that allow modification of pore size by varying experimental parameters such as air-flow, humidity and concentration.

PTs prefer to form highly ordered aggregates in thin film phase due to their high tendency to $\pi$ - $\pi$ stack their backbone. The result is polymer crystalline domains with improved charge mobility perpendicular to the stack. ${ }^{[27]}$ Increasing the length of alkyl side chain in PTs decreases the $\pi-\pi$ 


\section{WILEY-VCH}

stacking interaction and the crystallinity of the ordered phase, and this in turn decreases charge mobility across the polymer stack but increases side chain ordering ${ }^{[28]}$. Side chain length modification changes the interaction between polymer chains, hence affecting polymer aggregation behavior ${ }^{[29]}$. Stenzel et al. ${ }^{[15 a]}$ suggested that for amphiphilic polymers aggregation is a key condition in suppressing coalescence and leading to the formation of stable honeycomb films. PTs are also known to develop aggregation in mixed solvents where using such approach PT whiskers could be formed by ageing up to 2 months in Anisole solution ${ }^{[30]}$. In the present study we found that PTs have such aggregation behavior in solution even with the use of a good solvent, if the solution is left to age for 7-10 days. Therefore, we believe that for hydrophobic polymers like alkyl-substituted PTs, breath figure technique becomes successful if the polymer is dissolved in a good solvent and aged for a considerable period of time (days or even weeks). Such aggregates stop the coalescence and allow formation of stable ordered micro-droplets. We found that by removing these aggregates by filtration, for example by the use of a $0.45 \mu \mathrm{m}$ porous filter, the resulting solution was incapable of forming stable microporous structures.

During the processing of dynamic breath figure technique, the surface temperature is in the range of $-6^{\circ} \mathrm{C}$ to $0^{\circ} \mathrm{C}^{[8]}$. This low temperature arises due to evaporative cooling and makes the condensation of water droplets feasible at the solution-air interface. In this study, condensation is augmented by using a cold stage along with evaporative cooling. In the above defined temperature range, the polymer with the glass transition temperature $\left(T_{g}\right)$ lower but closest to the temperature of the substrate should easily form breath figures. Changing the side chain also changes polymer's $T_{g} \cdot{ }^{[11]}$ For PTs used in the present study, Table 2 lists $T_{g}$ values found in literature $^{[32]}$, according to which we now understand why P3DDT did not form large area honeycombs, given its very low $T_{g}$ of $-19^{\circ} \mathrm{C}$, a temperature unachievable during the evaporative cooling. Thus, amongst the polythiophenes considered in the present study, P3OT appears to 


\section{WILEY-VCH}

have the right combination of side chain length and $T_{g}$, the former helping in promoting aggregates in the aged solution of "perfect" size, the latter being closest to the surface temperature where condensation happens. The result is large area (over $5 \times 5 \mathrm{~mm}^{2}$ ) perfectly organized P3OT honeycombs. Both of these properties compete together and hence P3OT seems to have optimum side chain length to form most perfect breath figures out of the investigated PTs. The effect of side chain of polythiophenes on film morphology and optical properties has been studied widely in the bulk form ${ }^{[33]}$ as well as at the single polymer chain levels ${ }^{[19 a, 34]}$. Here, through optical and structural studies, we found clear differences in the polymer aggregation phase in the honeycomb framework, highly crystalline in the frame and a combination of amorphous and crystalline in the hole, with the ratio of amorphous to crystalline phases changing with the polymer side chain length.

\section{Conclusion}

Herein we provided a clear demonstration of how a relatively inexpensive process such as BFT can be realized as a repeatable method to produce micro-porous films from hydrophobic conjugated polymers, presenting for the first time semi-transparent honeycombs with area dimensions suitable for photovoltaic and sensory devices, with almost perfect organization of pores over sub-cm sized domains, which achievable by other methods like microlitoghraphy would be rather costly. Here we studied the effect of side chain length of PT on the breath figure forming capability. For PTs, a 6-carbon long side chain like for P3HT is known to provide optimal performance for photovoltaic applications, but we found in the present study that it is not best suited for honeycomb formation. Most probably, such short chain confers the polymer a high glass transition temperature which is unfavorable for aggregate formation. P3OT, the PT with a side chain length of 8 carbon chains (P3OT) is better for honeycomb formation. Using a 


\section{WILEY-VCH}

combination of optical and structural characterization methods, we demonstrated different polymer aggregation phases in the honeycomb framework, whose contributions change with the polymer side chain length.

One can envision semitransparent photovoltaic devices made out of breath figure polythiophene films doped with fullerenes or other semiconducting n-type nanomaterials or sensory devices based on such microporous films doped with energy transfer relevant nanoparticles. Since BFTbased microporous film offer a larger ( 140\%) surface area compared to a planar (dropcast/spincast film) due to its unique 3D structure, this in turn is expected to increase the efficiency of semitransparent photovoltaic devices or sensitivity in sensory devices incorporating such breath figure films.

\section{Materials and Methods}

\subsection{Materials and honeycomb film preparation by BFT.}

Commercially available polythiophenes were bought from Rieke Metals and carbon disulfide $\left(\mathrm{CS}_{2}\right)$ was from Sigma-Aldrich. All polymers have an average molecular weight of 70,000 to 90,000 with regio-regularity of approximately $96 \%$. Each of the four polymers was dissolved in $\mathrm{CS}_{2}$ with the help of magnetic stirring and stored in an airtight vial for ageing. Aged solutions (7 days on average) were drop casted using breath figure technique on glass or metal oxide surfaces and exposed to controlled humidity, temperature and airflow in order to preserve the order of honeycomb films. The films were made in ambient air and immediately kept into vacuum tight boxes after the deposition was complete. Dropcast samples of polymer solutions on glass substrates were exposed to laminar air flow with controlled humidity. In order to control the physical parameters of the experiment, a home built setup was used which is pictured in Figure 


\section{WILEY-VCH}

S1, SI. A glove box was connected to a humidifier and dehumidifier, which were controlled by a microcontroller to produce $\pm 1 \%$ relative humidity variation. A laminar flow control box was designed to control the airflow rate. Furthermore, the substrate holder was temperature controlled using water-cooled Copper plate. To compare the effect of side chain on honeycomb formation capability, all polythiophene polymers were cast using the following processing parameters:Concentration: $2 \mathrm{mg} / \mathrm{mL}$; Relative humidity: 65\%; Air flow rate: $2 \mathrm{~L} / \mathrm{min}$; substrate holder

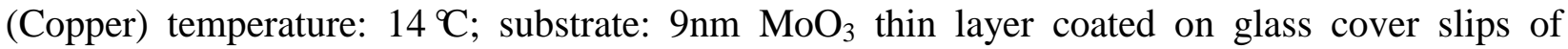
$0.17 \mathrm{~mm}$ thickness; volume of solution cast on substrate: $25 \mu \mathrm{L}$.

\subsection{Methods.}

Confocal FLIM and micro PL spectroscopy studies were carried out using a custom-built scanning-stage inverted microscope described elsewhere ${ }^{[18 b]}$. Samples were illuminated with 440 nm pulsed light from a solid state diode laser (LDH-440 Picoquant) using a 0.9 NA 60x dry objective lens (Olympus America). Fluorescence from the samples was collected by the same lens, filtered from laser excitation by a dichroic (532DRLP, Semrock) and by a band-pass filter (FF01-532RLP, Semrock), spatially filtered by a $5 \mu \mathrm{m}$ pinhole, and finally refocused onto a single-photon-counting avalanche photodiode (MPD, Picoquant). FLIM was carried on samples under nitrogen atmosphere to avoid photodegradation. FLIM images were acquired and analyzed with the commercial Symphotime 5.32 software (Picoquant). PL lifetimes reported in here were calculated as amplitude average lifetimes following double exponential fits. Micro PL spectra were acquired by a Spectra Pro 2300i spectrograph coupled to a back-illuminated CCD camera (Roper Scientific, PIXIS 100) by directing the collected fluorescence via a side port of the microscope and through a $5 \mu \mathrm{m}$ pinhole. Scanning electron microscopy (SEM) was performed with a Hitachi S-4800 SEM and with a 3nm Ag coating layer deposited on top of PT-based honeycomb films for improved conductivity. Small and Wide Angle X-ray scattering 


\section{WILEY-VCH}

(SAXS/WAXS) measurements were carried out on a Bruker-AXS Nanostar U instrument equipped with a $\mathrm{Cu}$ rotating anode, a Vantech 2000 detector and operated in the high-resolution configuration and short detector distance. The camera length for the instrument was calibrated using Silver Behenate. Polymer thin films were delaminated from the $\mathrm{MoO}_{3}$ coated glass substrates using $\mathrm{KOH}$ treatment and careful rinsing in DI water, SAXS/WAXS signals were recorded from free-standing films in transmission mode. Details on data analysis are given in SI.

\section{Supporting Information}

Supporting information includes details on BFT apparatus, photograph of a P3OT honeycomb film, pore size calculation, PL spectra for polymers in solution and drop cast thin film, details on WAXS data analysis and crystallinity value estimation. Supporting Information is available from the Wiley Online Library or from the author.

\section{Acknowledgements}

Research carried out at the Center for Functional Nanomaterials, Brookhaven National Laboratory and supported by the U.S. Department of Energy by Contract No. DE-SC0012704. Funding provided in part through a BNL/SBU seed grant and SMART Grid project (PKR and T.A.V)

Received: ((will be filled in by the editorial staff))

Revised: ((will be filled in by the editorial staff)) Published online: ((will be filled in by the editorial staff))

\section{REFERENCES}

[1] aA. Bolognesi, C. Mercogliano, S. Yunus, M. Civardi, D. Comoretto, A. Turturro, Langmuir 2005, 21, 3480-3485; bL. A. Connal, G. G. Qiao, Advanced Materials 2006, 18, 3024-3028.

[2] V. P. Shastri, I. Martin, R. Langer, Proc Natl Acad Sci U S A 2000, 97, 1970-1975.

[3] L.-S. Wan, Q.-L. Li, P.-C. Chen, Z.-K. Xu, Chemical communications 2012, 48, 44174419. 


\section{WILEY-VCH}

[4] aH. Yabu, M. Takebayashi, M. Tanaka, M. Shimomura, Langmuir 2005, 21, 3235-3237; bH. Yabu, M. Shimomura, Chemistry of Materials 2005, 17, 5231-5234; cM. Ma, R. M. Hill, Current Opinion in Colloid \& Interface Science 2006, 11, 193-202.

[5] aM. Yoshida, M. Asano, A. Safranj, H. Omichi, R. Spohr, J. Vetter, R. Katakai, Macromolecules 1996, 29, 8987-8989; bH. Bai, C. Du, A. Zhang, L. Li, Angewandte Chemie 2013, 52, 12240-12255.

[6] aT. Ohzono, T. Nishikawa, M. Shimomura, Journal of Materials Science 2004, 39, $2243-$ 2247; bM. Campbell, D. N. Sharp, M. T. Harrison, R. G. Denning, A. J. Turberfield, Nature 2000, 404, 53-56; cT. Cao, F. Wei, X. Jiao, J. Chen, W. Liao, X. Zhao, W. Cao, Langmuir 2003, 19, 8127-8129; dZ. Nie, E. Kumacheva, Nat Mater 2008, 7, 277-290.

[7] M. L. Hoa, M. Lu, Y. Zhang, Advances in colloid and interface science 2006, 121, 9-23.

[8] U. H. F. Bunz, Advanced Materials 2006, 18, 973-989.

[9] aD. Fritter, C. M. Knobler, D. A. Beysens, Physical Review A 1991, 43, 2858-2869; bA. Steyer, P. Guenoun, D. Beysens, C. M. Knobler, Physical Review B 1990, 42, 1086-1089.

[10] M. Hernandez-Guerrero, M. H. Stenzel, Polymer Chemistry 2012, 3, 563-577.

[11] aM. H. Stenzel, C. Barner-Kowollik, T. P. Davis, Journal of Polymer Science Part A: Polymer Chemistry 2006, 44, 2363-2375; bL. S. Wan, L. W. Zhu, Y. Ou, Z. K. Xu, Chemical communications 2014, 50, 4024-4039; cP. Escalé, L. Rubatat, L. Billon, M. Save, European Polymer Journal 2012, 48, 1001-1025; dA. Muñoz-Bonilla, M. Fernández-García, J. Rodríguez-Hernández, Progress in Polymer Science 2014, 39, 510554.

[12] H. Battenbo, R. J. Cobley, S. P. Wilks, Soft Matter 2011, 7, 10864.

[13] G. Widawski, M. Rawiso, B. Francois, Nature 1994, 369, 387-389.

[14] J. Peng, Y. C. Han, Y. M. Yang, B. Y. Li, Polymer 2004, 45, 447-452.

[15] aM. H. Stenzel, Australian Journal of Chemistry 2002, 55, 239-243; bX. Y. Zhao, Q. Cai, G. X. Shi, Y. Q. Shi, G. W. Chen, Journal of Applied Polymer Science 2003, 90, 18461850; cT. Nishikawa, J. Nishida, R. Ookura, S.-I. Nishimura, V. Scheumann, M. Zizlsperger, R. Lawall, W. Knoll, M. Shimomura, Langmuir 2000, 16, 1337-1342; dO. Karthaus, X. Cieren, N. Maruyama, M. Shimomura, Materials Science \& Engineering CBiomimetic and Supramolecular Systems 1999, 10, 103-106; eA. Böker, Y. Lin, K. Chiapperini, R. Horowitz, M. Thompson, V. Carreon, T. Xu, C. Abetz, H. Skaff, a. D. Dinsmore, T. Emrick, T. P. Russell, Nat Mater 2004, 3, 302-306.

[16] aJ. Y. Ding, A. J. Zhang, H. Bai, L. Li, J. Li, Z. Ma, Soft Matter 2013, 9, 506-514; bA. Zhang, C. Du, H. Bai, Y. Wang, J. Wang, L. Li, ACS Applied Materials \& Interfaces 2014, 6, 8921-8927.

[17] aJ. Ding, J. Gong, H. Bai, L. Li, Y. Zhong, Z. Ma, V. Svrcek, Journal of colloid and interface science 2012, 380, 99-104; bL. A. Connal, R. Vestberg, C. J. Hawker, G. G. Qiao, Macromolecules 2007, 40, 7855-7863.

[18] aM. H. Nurmawati, R. Renu, P. K. Ajikumar, S. Sindhu, F. C. Cheong, C. H. Sow, S. Valiyaveettil, Advanced Functional Materials 2006, 16, 2340-2345; bH. Tsai, Z. Xu, R. K. Pai, L. Wang, A. M. Dattelbaum, A. P. Shreve, H.-L. Wang, M. Cotlet, Chemistry of Materials 2011, 23, 759-761; cL. Song, R. K. Bly, J. N. Wilson, S. Bakbak, J. O. Park, M. Srinivasarao, U. H. F. Bunz, Advanced Materials 2004, 16, 115-118; dY. Lu, L. Wang, B. Zhao, G. Xiao, Y. Ren, X. Wang, C. Li, Thin Solid Films 2008, 516, 6365-6370; eB. Erdogan, L. Song, J. N. Wilson, J. O. Park, M. Srinivasarao, U. H. F. Bunz, Journal of the American Chemical Society 2004, 126, 3678-3679. 


\section{WILEY-VCH}

[19] aT. Adachi, J. Brazard, R. J. Ono, B. Hanson, M. C. Traub, Z.-Q. Wu, Z. Li, J. C. Bolinger, V. Ganesan, C. W. Bielawski, D. A. Vanden Bout, P. F. Barbara, Journal of Physical Chemistry Letters 2011, 2, 1400-1404; bI. F. Perepichka, D. F. Perepichka, H. Meng, F. Wudl, Advanced Materials 2005, 17, 2281-2305; cM. Jaymand, M. Hatamzadeh, Y. Omidi, Progress in Polymer Science 2015.

[20] A. Okabe, B. Boots, K. Sugihara, S. N. Chiu, Spatial Tesselations: Concepts and Applications of Voronoi Diagrams, 2nd Edition ed., John Wiley \& Sons Ltd, 2000.

[21] A. J. Krejci, C. G. W. Thomas, J. H. Dickerson, Phys Rev E 2013, 87.

[22] aH. Jiang, P. Taranekar, J. R. Reynolds, K. S. Schanze, Angewandte Chemie International Edition 2009, 48, 4300-4316; bZ. Xu, H. Tsai, H.-L. Wang, M. Cotlet, The Journal of Physical Chemistry B 2010, 114, 11746-11752.

[23] aP.-T. Huang, Y.-S. Chang, C.-W. Chou, Journal of Applied Polymer Science 2011, 122, 233-240; bF. Wang, G. C. Bazan, Journal of the American Chemical Society 2006, 128, 15786-15792.

[24] aJ. Clark, C. Silva, R. H. Friend, F. C. Spano, Physical Review Letters 2007, 98, 206406; bC. Carach, M. J. Gordon, The Journal of Physical Chemistry B 2013, 117, 1950-1957.

[25] aX. M. Jiang, R. Österbacka, C. P. An, Z. Valy Vardeny, Synthetic Metals 2003, 137, 1465-1468; bG. Rumbles, I. D. W. Samuel, L. Magnani, K. A. Murray, A. J. DeMello, B. Crystall, S. C. Moratti, B. M. Stone, A. B. Holmes, R. H. Friend, Synthetic Metals 1996, 76, 47-51.

[26] Y.-Z. Ma, R. W. Shaw, X. Yu, H. M. O’Neill, K. Hong, The Journal of Physical Chemistry B 2012, 116, 14451-14460.

[27] R. Noriega, J. Rivnay, K. Vandewal, F. P. V. Koch, N. Stingelin, P. Smith, M. F. Toney, A. Salleo, Nature Materials 2013, 12, 1038-1044.

[28] aY. D. Park, D. H. Kim, Y. Jang, J. H. Cho, M. Hwang, H. S. Lee, J. A. Lim, K. Cho, Organic Electronics 2006, 7, 514-520; bR. J. Kline, M. D. McGehee, E. N. Kadnikova, J. Liu, J. M. J. Fréchet, Advanced Materials 2003, 15, 1519-1522.

[29] T. Yamamoto, D. Komarudin, M. Arai, B.-L. Lee, H. Suganuma, N. Asakawa, Y. Inoue, K. Kubota, S. Sasaki, T. Fukuda, H. Matsuda, Journal of the American Chemical Society 1998, 120, 2047-2058.

[30] M. He, J. Ge, M. Fang, F. Qiu, Y. Yang, Polymer 2010, 51, 2236-2243.

[31] S. Malik, A. K. Nandi, Journal of Polymer Science Part B: Polymer Physics 2002, 40, 2073-2085.

[32] aR. Payerne, M. Brun, P. Rannou, R. Baptist, B. Grévin, Synthetic Metals 2004, 146, 311 315; bS. Pal, S. Roy, A. K. Nandi, J Phys Chem B 2005, 109, 18332-18341.

[33] aH.-J. Wang, L.-H. Chan, C.-P. Chen, S.-L. Lin, R.-H. Lee, R.-J. Jeng, Polymer 2011, 52, 326-338; bW. Y. Huang, C. C. Lee, S. G. Wang, Y. K. Han, M. Y. Chang, Journal of The Electrochemical Society 2010, 157, B1336-B1342.

[34] M. Vacha, S. Habuchi, Npg Asia Materials 2010, 2, 134-142. 


\section{WILEY-VCH}

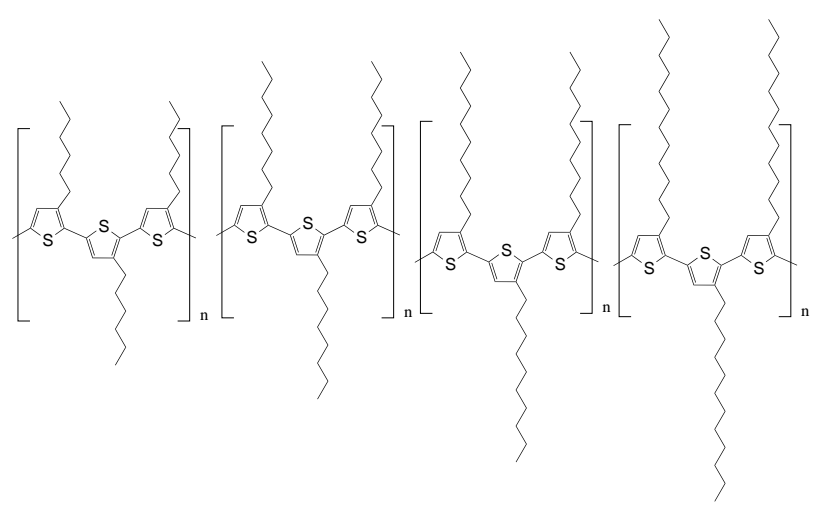

Scheme 1. Polythiophenes with varying side chain length: poly (3-hexyl thiophene), P3HT; poly (3-Octyl thiophene), P3OT; poly (3-Decyl thiophene), P3DT; Poly (3-Dodecyl thiophene), P3DDT.
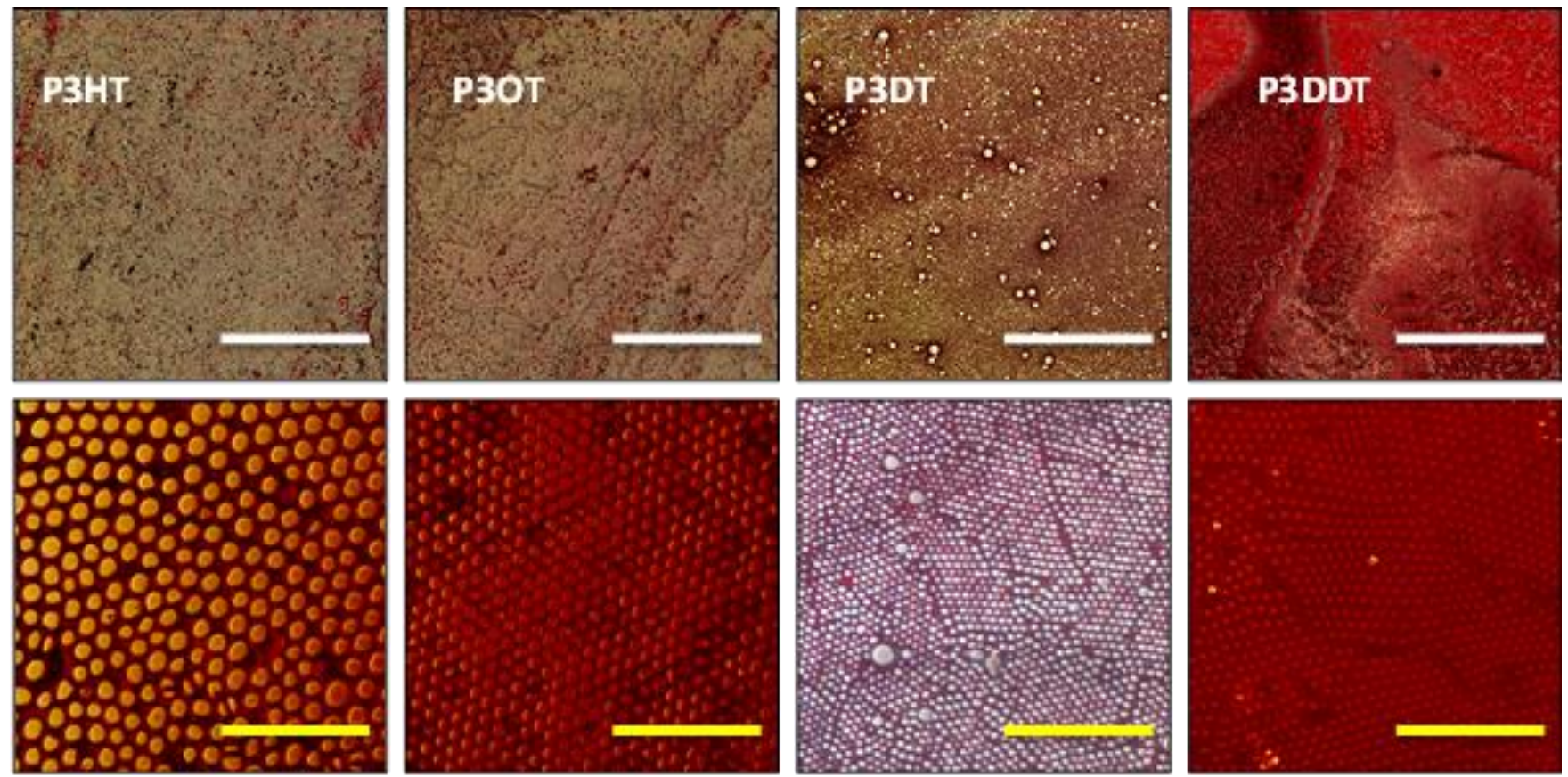

Figure 1. Optical (transmission) micrographs of PT honeycomb thin films showing long range ordered microporous structures. Top: low magnification; Bottom: high magnification. Scale bars are $400 \mu \mathrm{m}$ (left: white) and $40 \mu \mathrm{m}$ (right: yellow). 


\section{WILEY-VCH}
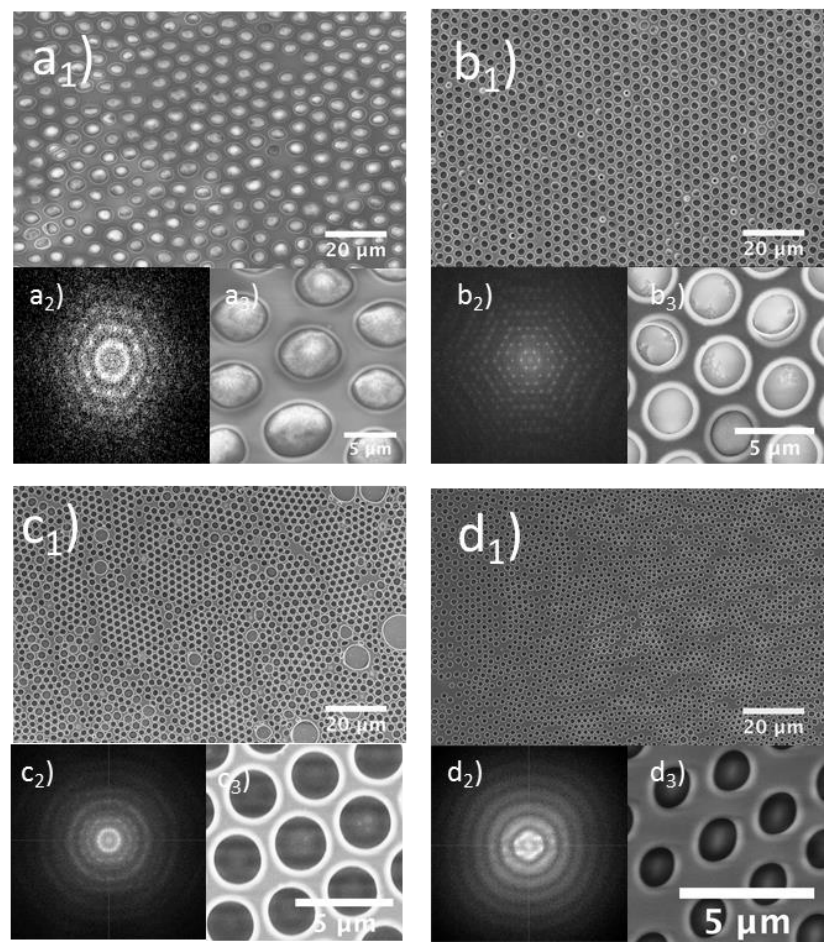

Figure 2. Scanning Electron Micrographs of PT-based honeycomb films: a) P3HT b) P3OT c) P3DT d) P3DDT; Subscript 2 for each image shows the FFT pattern calculated after converting the image. Subscript 3 shows zoomed in image of single hexagonal lattice for each image.

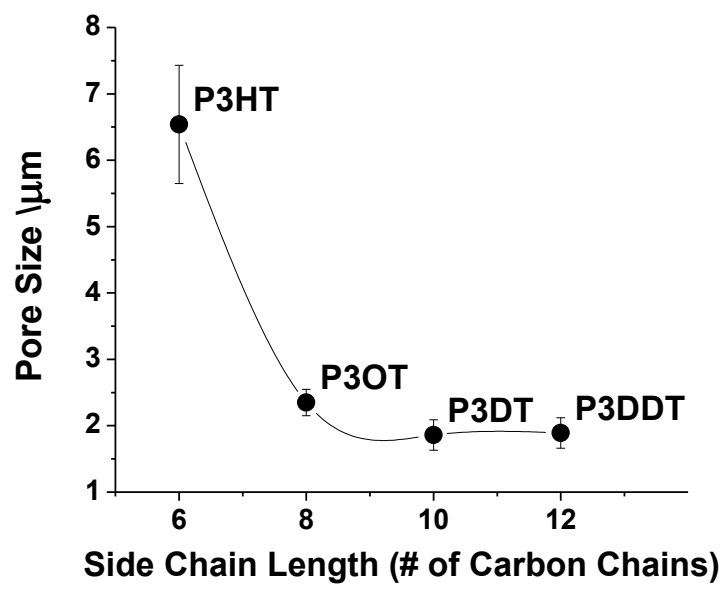

Figure 3. Pore size vs side chain length for PT-based honeycombs. 


\section{WILEY-VCH}
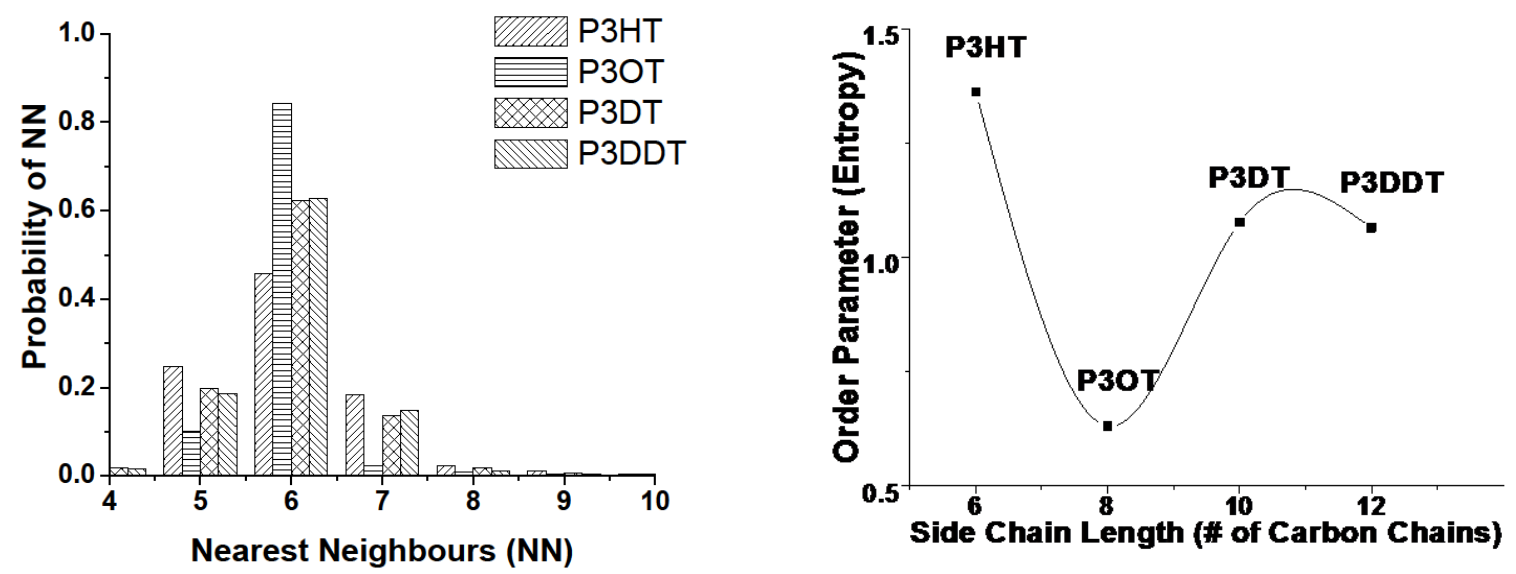

Figure 4. left) Probability distribution of nearest neighbors in honeycomb films determined by Voronoi Tessellation method. Right) Order parameter (entropy, S) calculated using Nearest Neighbor probability distribution. P3OT shows the lowest value of $\mathrm{S}$, hence exhibits the most ordered film. 


\section{WILEY-VCH}
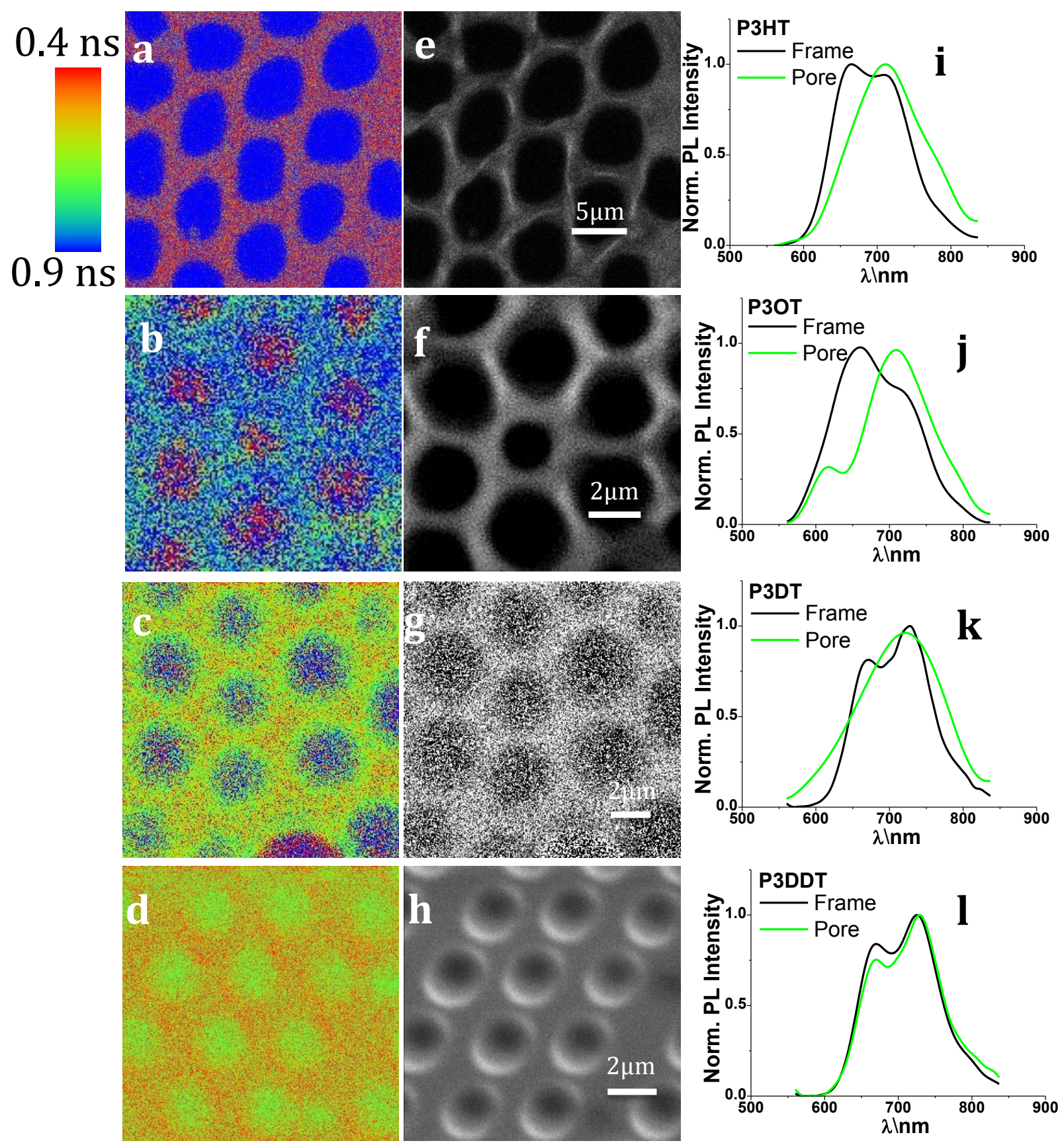

Figure 5. Confocal FLIM (a-d), PL Intensity image (e-h) and micro PL spectroscopy (i-l) of honeycomb thin films processed from: (a, e, i) P3HT, (b, f, j) P3OT, (c, g, k) P3DT and (d, h, l) P3DDT. Micro PL spectra were recorded at the frame (black color) and in the pore (green color) of the honeycomb cell units. 


\section{WILEY-VCH}
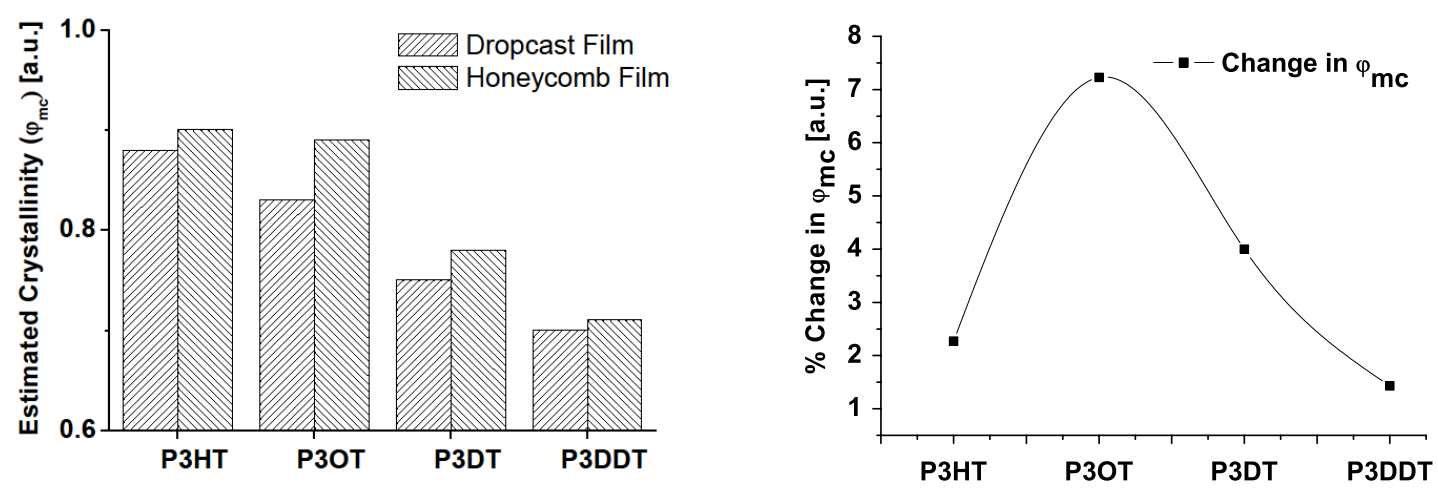

Figure 6. Left: crystallinity values $\left(\varphi_{\mathrm{mc}}\right)$ obtained from WAXS measurements of dropcast and honeycomb PT-based thin films; right: percent change in crystallinity value, $\varphi_{\mathrm{mc}}$, from dropcast to honeycomb PT-based thin films.

Table 1. PL parameters for solution, drop-cast and honeycomb samples as derived from Figure 5 and S3, Supplementary Information.

\begin{tabular}{|c|c|c|c|c|c|c|c|c|}
\hline \multirow{2}{*}{ sample } & \multicolumn{2}{|c|}{ P3HT } & \multicolumn{2}{c|}{ P3OT } & \multicolumn{2}{c|}{ P3DT } & \multicolumn{2}{c|}{ P3DDT } \\
\cline { 2 - 9 } & $\tau_{\mathrm{PL}} / \mathrm{ns}$ & $\mathrm{R}_{\mathrm{PL}}$ & $\tau_{\mathrm{PL}} / \mathrm{ns}$ & $\mathrm{R}_{\mathrm{PL}}$ & $\tau_{\mathrm{PL}} / \mathrm{ns}$ & $\mathrm{R}_{\mathrm{PL}}$ & $\tau_{\mathrm{PL}} / \mathrm{ns}$ & $\mathrm{R}_{\mathrm{PL}}$ \\
\hline solution & 0.58 & - & 0.58 & - & 0.58 & - & 0.58 & - \\
\hline Dropcast & 0.47 & 1.16 & 0.42 & 1.0 & 0.72 & 1.15 & 0.86 & 1.04 \\
\hline $\begin{array}{c}\text { honeycomb } \\
\text { frame }\end{array}$ & 0.75 & 1.06 & 0.42 & 1.3 & 0.81 & 0.8 & 0.64 & 0.84 \\
\hline $\begin{array}{c}\text { honeycomb } \\
\text { hole }\end{array}$ & 0.62 & 0.65 & 0.49 & 0.33 & 0.70 & - & 0.60 & 0.75 \\
\hline
\end{tabular}

${ }^{*} \tau_{\mathrm{PL}}$, intensity averaged PL lifetime; $\mathrm{R}_{\mathrm{PL}}, \mathrm{PL}$ intensity ratio for high and low energy peaks in the PL spectrum. 


\section{WILEY-VCH}

Table 2: glass transition temperatures of Polythiophene derivatives. ${ }^{[32]}$

\begin{tabular}{|c|c|}
\hline Polymer & $\boldsymbol{T}_{\boldsymbol{g}}$ \\
\hline P3HT & $20.3^{\circ} \mathrm{C}$ \\
\hline P3OT & $-9.2^{\circ} \mathrm{C}$ \\
\hline P3DT & -- \\
\hline P3DDT & $-19^{\circ} \mathrm{C}$ \\
\hline
\end{tabular}




\section{WILEY-VCH}

\section{Table of Contents}

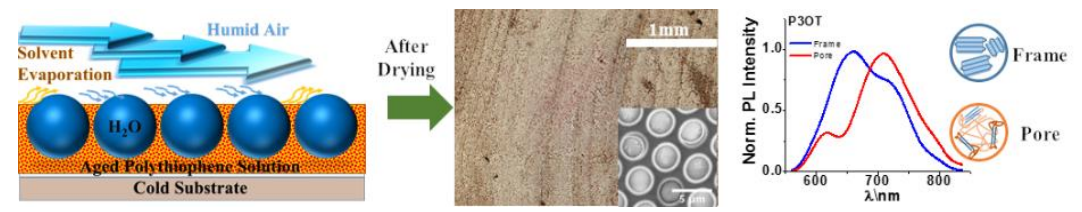

Ordered microporous thin films of centimeter sized large area were successfully prepared from commercially available polythiophenes by the use of Breath Figure Technique. Structural and optical characterization of these thin films reveals increased crystallinity and ordered aggregates in the frame. 